\title{
Representation-saving effects of prior knowledge in memory for simple technical prose
}

\author{
WALTER JOHNSON and DAVID KIERAS \\ University of Arizona, Tucson, Arizona
}

\begin{abstract}
Prior knowledge of the content of a passage should reduce the effort required to encode the passage and facilitate its recall. This paper presents such effects of prior knowledge upon comprehension and memory of simple technical prose. The basic procedure was to collect ratings of the amount of prior knowledge for individual passage sentences and individual subjects, and then to determine whether these familiarity ratings predicted study time and recall. In order to validate the rating method, and also to obtain data on prior knowledge of individual facts, subjects also completed an objective test of prior knowledge of the passage facts. Three different encoding-task conditions were used: a self-paced study task, a forced-pace study task, and an incidental-learning task. A cued-recall test followed each condition. In the self-paced task, readers studied unfamiliar material longer than familiar material, but recalled at the same level regardless of familiarity. In contrast, familiarity did predict recall in the forced-pace and incidental tasks. This task specificity is explained in terms of the subjects' encoding strategies. The basic effect of prior knowledge can be explained not only by the elaboration principle, but also by a representation-saving principle, which is presented in a simulation model that can account for the effects of familiarity on study time.
\end{abstract}

We all have the strong intuition that a person should be able to learn and remember material better if the person has a lot of prior knowledge about the content of the material. This issue has great practical importance in understanding how people learn technical material from textbooks and how they use technical documents such as maintenance manuals. However, the experimental literature documenting the relationship between content familiarity and prose memory performance is sparse, and the available results are somewhat contradictory. There are few studies presenting direct evidence that, in a prose memory situation, prior knowledge of the subject matter facilitates study and memory for passage content. Chiesi, Spilich, and Voss (1979) and Spilich, Vesonder, Chiesi, and Voss (1979) found that subjects who were very familiar with the terminology, rules, and procedures of baseball recalled important facts about a baseball game better than did subjects who were relatively unfamiliar with baseball. Anderson (1981) found that subjects given prior knowledge about individuals learned new information about the individuals faster, but retrieved this information more slowly. Graesser, Hoffman, and Clark (1980) found a slight facilitative effect of prior knowledge on reading times,

The order of authors was determined at random. This research was supported by the Office of Naval Research, Personnel and Training Research Programs, under Contract N00014-816-0699, Contract Authority Identification NR-157-473, awarded to the second author. The experiment reported is a portion of Walter Johnson's master's thesis. Requests for reprints should be addressed to David Kieras, Department of Psychology, University of Arizona, Tucson, Arizona 85721. but in another experiment, Graesser, Hauft-Smith, Cohen, and Pyles (1980) found that prior knowledge can in fact have a detrimental effect on recall.

\section{Mechanisms of Prior Knowledge}

Despite the lack of experimental study of prior knowledge, the theoretical issues are very clear. There are three explanations for why prior knowledge should facilitate study and recall of prose material.

Schema transfer. One approach is that if a reader has the appropriate schema for the passage content, facilitation of study, as well as retrieval, should occur. If a schema is an organized knowledge structure that guides perception (Rumelhart, 1980), it should reduce encoding time. Since the structure for encoding the material is already present, the reader can simply instantiate the schema with the to-be-encoded material. Remembering from a schematic knowledge base can be viewed in much the same way. Rumelhart and Ortony (1977) suggest that remembering can be thought of as perceiving, with memory as the modality. In this way a schema could also be a guide for recall. Hence, prior knowledge of a relevant schema should facilitate prose memory performance (see Graesser, Hoffman, \& Clark, 1980).

But the relevancy of schematic knowledge to technical prose has not yet been established, and it can be argued that schemata have only a weak role in technical prose (Kieras, in press-c). Schemata are usually described in terms of stereotypical sequences of events, which appear in simple stories (e.g., Graesser, 1981), goal-directed activities (e.g., Chiesi, Spilich, \& Voss, 1979; Spilich et al., 1979), or the plot structure of a story, indepen- 
dent of passage content (e.g., Thorndyke, 1977). These types of prose are quite different from technical prose, which is often simply an exposition of facts about a subject and which often does not include a temporal sequence of events or a goal. Thus, it appears that schematic knowledge is not necessarily applicable to technical prose. This conclusion is supported by Graesser, Hoffman, and Clark (1980), who found that rated narrativity strongly predicted recall of passages and that technical passages were very low on this dimension.

Elaboration. Another theoretical approach to explaining the role of prior knowledge is the concept of elaboration (Anderson \& Reder, 1979). During study, additional relationships interconnecting the concepts are inferred and are added to the memory structure, resulting in alternate retrieval paths that facilitate recall. Familiar material should be easier to encode and remember than unfamiliar material because there is information already present in memory to serve as a basis for elaboration. Furthermore, highly familiar information may already have many alternate retrieval paths present, since the concepts involved would already be highly interconnected in the memory structure. Thus, the effort and time required to perform elaborative processing on familiar material should be less than for unfamiliar material.

There is support in the literature for effects of prior knowledge on elaboration (see Gagne, 1978). For example, Johnson (1973) found that linguistic units rated high on meaningfulness were recalled better than those rated low on this dimension. Meaningfulness was measured as in the traditional verbal learning measure $m$, that is, the ease with which a linguistic unit could call forth associations with past experience. Assuming that meaningfulness is a measure of prior knowledge, it appears that recall is aided by preexisting knowledge. However, in Johnson's study, subjects were instructed to rate phrases that aroused sensory imagery as being highly meaningful. But the memory effects related to $\mathrm{m}$ value have been shown to be due to imagery value (Paivio, 1971). Thus, the better recall for highly meaningful prose material may actually be an effect of imagery value. Hence, although elaboration is a possible explanation for the role of prior knowledge in facilitating recall, this mechanism has not yet been directly demonstrated.

Representation saving. Another explanation, offered here, is that prior knowledge works on a propositionby-proposition basis by saving on the amount of representation building performed during comprehension. That is, if the reader already knows an individual proposition, the encoding effort to put it into memory is either unnecessary or greatly reduced. In more detail, before an input proposition is stored in long-term memory, a check is done to see if the proposition is already present. If so, then the memory representation for the proposition does not need to be constructed again; rather, the preexisting representation only needs to be tagged to represent its appearance in the specific passage. In contrast, if an input proposition is unknown, a representation for it has to be built from "scratch," and then tagged. Such mechanisms have been used in several simulation models of comprehension and memory, such as those of Anderson and Bower (1973) and Kieras (1977). This hypothesis yields the straightforward prediction that some measure of encoding effort, such as study time in a self-paced presentation situation, should be related to the number of passage propositions that the reader already knows. Furthermore, propositions aiready known should be recalled better, since they would require less encoding effort for successful recall.

\section{Methodological Approach}

Despite the obviousness of the predicted effects of prior knowledge, demonstrating them presents some severe methodological problems, which perhaps accounts for the unclear state of the literature. It would seem that, in the ideal experiment, the amount of prior knowledge possessed by the subject would be manipulated experimentally by pretraining. But, as argued below, this approach suffers because experimentally acquired knowledge does not have the same properties as true preexperimental knowledge. Another obvious approach would be comparing performance on materials that are rated high and low in content familiarity. But this would ignore the considerable amount of betweensubject variation in background knowledge and would discard much of the information in the familiarity variable. Eliminating certain obvious confounds would also be extremely hard; for example, material that is generally less familiar can easily involve longer sentences and rarer words.

To avoid these problems, a quasi-experimental approach based on multiple-regression analysis was used in the study reported here. In essence, prior knowledge was manipulated by choosing materials that varied widely in their familiarity to typical subjects, and by allowing the sampling of subjects to further vary the familiarity at the level of individual subjects. The statistical question is then whether performance can be predicted from prior knowledge, with the regression analysis providing statistical control for confounded properties of the material, such as word frequency and sentence length. In order to make use of the fact that individual subjects differ in their knowledge, an assessment of prior knowledge can be obtained from each subject, along with the performance measures of study time and recall. These variables can then be related to each other at the level of individual subjects.

An especially important feature of the approach concerns whether the desired prior-knowledge effect appears within, rather than between, subjects. This is important because there could be general correlations in the subject population that mimic the desired withinsubject effect of prior knowledge. For example, an apparent correlation between study time and familiarity could be due simply to the fact that subjects who are 
more knowledgeable are faster readers, and not to any interesting theoretical mechanism involving prior knowledge. One approach to eliminating this possibility is to equate subjects on reading ability (e.g., Chiesi et al., 1979; Spilich et al., 1979). But the approach used here ensured that the variation in familiarity had a large within-subject component. Furthermore, analyses were performed in which the between-subject variation was removed statistically, such that only within-subject variation was present (see Johnson \& Kieras, Note 1). Then, if a prior-knowledge effect was still present, it would have to be a within-subject effect.

\section{Pilot Studies}

Some pilot studies whose methods and results were the basis for this study on prior knowledge are summarized here. In the first pilot study, subjects were asked to rate how much of the information in each of a set of passages they knew before the experiment, and their study time and recall were measured. The study times decreased with increasing rated familiarity; the correlation using the familiarity and reading times for each subject on each passage was $-.19, \mathrm{p}<.05$, which rose to $-.46, \mathrm{p}<.01$, when a within-subject $\mathrm{z}$ transform of the reading times was used to eliminate differences in subject reading baseline and rate. However, there was an apparent confounding of overall familiarity with sentence complexity. The very familiar passages tended to have simpler sentence structure than the less familiar ones.

A second pilot study attempted to compensate for this confounding by manipulating the amount of prior knowledge, keeping the passages constant. The subjects first studied familiarization passages, and then the next day studied, rated, and recalled test passages. The content of the familiarization passages overlapped (in paraphrase) to different extents with the content of the test passages. Greater overlap increased the knowledge ratings on the test passages, reduced the study time, and increased recall. Since the test passages remained constant, it appears that the confounding in the first study was ruled out as an explanation. However, the facilitation was not in direct proportion to the amount of overlap between the familiarization and the test passages. Certain features of the results and materials, features that cannot be discussed here due to space limitations, suggested that this was due to the subjects' remembering the familiarization information in terms of the macrostructure (Kintsch \& van Dijk, 1978) of the specific passages, rather than as individual facts. Thus, the transfer of knowledge between passages appeared to be governed by the relationship between the passage macrostructures, rather than operating at the level of simple transfer of individual propositions. Clearly, an important feature of true prior knowledge is that the individual facts can be used independently of the prose context in which they were originally learned. Thus, attempting to install specified prior knowledge in subjects by using transfer designs may result in forms of knowledge rather different from genuine preexperimental prior knowledge. Other recent results in the literature (e.g., Anderson, 1981) have supported the same conclusion.

A third pilot experiment (see Johnson \& Kieras, Note 1) used a set of passages in a self-paced onesentence-at-a-time presentation procedure, and measures of rated familiarity, study time, and recall were obtained at the level of individual subjects' processing of individual sentences. The subjects read each passage twice, first, in order to rate how much of each sentence content they knew prior to the experiment, and second, in order to study for later recall. The question was whether rated familiarity would predict study time and recall, with other variables being statistically controlled by a regression analysis. Having subjects read each passage twice may appear to be a questionable procedure, in that two study trials might weaken the effect of familiarity. However, the familiarity rating was only weakly correlated with the time taken to make the rating, but was definitely correlated with the study time. This suggests that subjects did not deliberately study the sentences while rating them. Finally, although the effect of prior knowledge might be weakened by two presentations, the advantages of this within-subject measurement of prior knowledge and performance justified the procedure.

Effects of prior knowledge were obtained for both study time and recall, but the recall effect was extremely weak. A straightforward explanation for this result is that subjects could have used a strategy of adjusting their study time based on sentence familiarity in order to achieve a desired level of recall. Thus, subjects did not study all of the sentences for the same amount of time, but, rather, studied unfamiliar sentences for longer periods of time and then recalled all material at about the same level. The result is a definite study-time effect, about $.8 \mathrm{sec}$ less time for each point on the 1.7 familiarity scale, but a very weak recall effect of about .7\% more recall for each point on the scale.

\section{EXPERIMENT}

The present experiment was designed to confirm the hypothesis suggested by the last of the pilot experiments. If subjects were adjusting their study times on the basis of familiarity in order to obtain a constant recall level, then, with study time held constant, recall would be expected to depend on familiarity. Alternatively, if subjects did not know that they would be tested for later recall, they would not adjust their study times, and therefore familiarity effects on recall also would be expected to appear.

Thus, familiarity effects were studied in three conditions: a self-paced condition, in which the subjects studied each sentence as long as desired; a forced-pace condition, with study time held at a predetermined level 
for each subject and each sentence; and an incidental condition, in which the subjects did not know they were to recall the passages later. In the forced-pace condition, the amount of study time allowed on a sentence was the time that the same subject had taken to rate the same sentence for content familiarity. This provided a simple way to adjust the permitted study time to an amount tailored to both individual sentences and individual subjects. Using the rating time was justified since, as described above, in the pilot experiment the time to produce ratings was only slightly related to the rating itself. Thus, the rating time conveniently provided a measure of how long it took to read and comprehend a sentence, but was not long enough to allow much memorization of an unfamiliar sentence. The subjects in the incidental-learning task did not study the sentences after rating them for content familiarity, and were not told there would be a final recall test. Instead, they rated the sentences for the importance of each sentence to the subject's perceived main idea of the passage, and then were given the recall test. This measure of importance was also used as a predictor variable.

In the pilot experiments, familiarity was assessed simply by having subjects rate their prior knowledge of each sentence on a 7 -point scale. This method seems to be a logical choice for assessing an individual's familiarity with the content of a sentence, but it has not been validated. This experiment included an objective multiplechoice test designed to give an assessment of prior knowledge at the level of a subject's knowledge of the individual propositions in the passages. This measurement could then be compared with the familiarity ratings, and also allowed the recall effects of prior knowledge to be examined at the level of individual propositions.

\section{Method}

Materials. The passages were based on the four studied in Kieras (1982). They were all modified to be eight sentences long. An example appears in Table 1; all four may be found in Johnson and Kieras (Note 1). The passages were designed to vary in familiarity both within and between passages. A propositional analysis was prepared for each passage, based on Bovair and Kieras (Note 2). For each passage, a keyword was selected as a recall cue. An additional passage was prepared for use as a practice passage.

The knowledge test was carefully constructed to test for knowledge of specific propositions but to not give the subject much information in the course of taking the test. The test contained 82 multiple-choice questions; the first 2 were practice questions. Fifty-five of the questions tested for one or more specific propositions, and the remaining questions were fillers. It was possible to test for knowledge of only $66 \%$ of the passage propositions, because it was necessary to state some propositions in the questions and because other propositions were essentially impossible to test without stating an excessive number of other propositions.

Design. The three task conditions were a between-subject manipulation. Each subject completed the knowledge test and then processed all four passages, thus providing a familiarity rating for each sentence, a study time on each sentence, and recall for each passage. Thus, each subject contributed both the familiarity variables and the performance variables. The order of presentation of the passages was randomized for each subject, but this order was the same for the rating, study, and recall portions of the experiment for each subject.

Subjects. Ninety-two male and female undergraduates at the University of Arizona were each paid $\$ 5.00$ for their participation. The subjects were recruited by an ad in the student newspaper and by signs posted in campus buildings. Two subjects had to be dropped because they wrote their recall at the wrong point in the experiment. Thus, there were 30 subjects in each task condition.

Apparatus and Procedure. A laboratory computer driving a set of three video terminals was used to present all stimuli and to collect responses and response latencies. However, the subjects wrote their recall on notepads by hand.

The subjects were run in groups of one to three, with each group being assigned a condition in a haphazard way that kept the number of subjects in each group roughly equal. All subjects first performed the knowledge test, in which each multiplechoice test question was displayed on the video terminal and the subjects typed in the answer. Then the subjects rated on a 1-7 scale each passage sentence for prior knowledge. The subjects were instructed to choose a rating based on how much of the information in the sentence they knew before the experiment. At this point in the experiment, the subjects had not been told that later recall would be required.

When the subjects finished the rating task, they were given the next instructions, which depended on the condition. The self-paced subjects were told that they should study each sentence for as long as desired and that they would recall the passages later. Each sentence was presented on the terminal screen, and the subjects tapped the space bar to change the display to the next sentence in the passage. The computer recorded the time each sentence was left on the screen as the study time for that sentence. The forced-pace subjects were instructed that the sentences would appear on the screen at a predetermined rate, that they might not be able to study the sentences for as long as they would like, and that they would have to recall the passages later. When the subjects indicated their readiness by tapping the space bar, the sentences appeared on the screen one at a time at a predetermined rate. The time each sentence was displayed was the time that the very same subject had taken to provide the familiarity rating for the same sentence earlier in the experiment. The incidental-condition subjects were not told that they would have to recall the passages later. The subjects were instructed to try to discover the main idea of the passage and, as they went along, to rate on a

Table 1

Example Passage

1. Throughout history, man has used metals for many purposes.

2. The ancient Hellenes used bronze swords.

3. The Hellenes invaded ancient Greece before the Trojan war.

4. The Incas lived in South America.

5. The Incas used gold in religious ceremonies.

6. The Spaniards conquered the Incas for their gold.

7. Aluminum is used in camping equipment.

8. Titanium is the brilliant white pigment used in oil paints that are used by some artists. 
1.7 scale the importance of each sentence to the main idea (see Kieras, 1982, in press-c).

After studying or rating all of the passages, the subjects began the recall task. The instructions specified that they were to write all they could remember about each passage, but their recall did not have to be verbatim. The computer displayed a keyword from each passage as a cue for recall, one at a time and in the same order as the passages had been seen in the rating and study tasks. The subjects wrote their complete recall of the passage before tapping the space bar to obtain the next cue.

\section{Results}

There are three components to the results: (1) The recall of individual propositions was analyzed in terms of the prior knowledge of individual propositions, as assessed by the knowledge test. (2) Since the familiarity ratings were obtained for whole sentences, the proportion of propositions recalled for whole sentences was related to the familiarity ratings. (3) The study time for the sentences was also related to the familiarity ratings. Study time was analyzed only in the self-paced task conditions, because this was the only true study-time measure. Study times in the forced-pace condition were necessarily the same as the rating times, and study time in the incidental condition is not a true "study time," but rather is the time taken to rate a sentence for importance.

In scoring the knowledge test, each subject received credit for knowing a specific proposition if he or she correctly answered the test question testing for that proposition. In the case of questions that tested for more than one proposition, credit was given for all of the propositions. The resulting scores specified the individual propositions that were known and that were not known by each subject. Also, the proportion of propositions known by each subject in each passage sentence was determined, with only the propositions explicitly tested being considered. The proportion of known propositions in the sentences correlated $(\mathrm{r}=.66)$ with the familiarity ratings for the same sentences. Thus, the familiarity rating is valid as a measure of actual prior knowledge, but it is influenced to some extent by factors other than prior knowledge (see Johnson \& Kieras, Note 1, for more detail).

The recall protocols were scored blindly, without knowledge of the original task conditions. Each protocol was scored independently by two judges, and a third judge then reconciled any discrepancies between the two scores. The scoring was done in the usual propositionbased fashion, in which credit is given for reproduction of individual propositions. The scoring criterion was strict: Synonymous terms were permitted, but otherwise exact reproductions of the passage propositions were required (see Bovair \& Kieras, Note 2). The specific propositions recalled by each subject were recorded, and the proportion of propositions recalled by each subject on each sentence was determined.

The variables used in the regression analyses described below were as follows: the proportion of propositions recalled per sentence (RECALL), familiarity rating
(FAMILIARITY), study time (STUDYTIME), the mean Standardized Frequency Index values (taken from Carroll, Davies, \& Richman, 1971) for the content words in the sentence (FREQUENCY), the proportion of propositions known in the sentence according to the knowledge test (KNOWLEDGE), and the number of words in the sentence (WORDS). The mean importance rating of each sentence, collected from subjects in the incidental task, was used as a predictor variable for all conditions (IMPORTANCE). Since the first sentence in a passage usually requires a disproportionately large reading time, and of ten plays a special role (see Kieras, 1981 a, 1982, in press-c), a dummy variable (see Cohen \& Cohen, 1975), called FIRST, was defined to indicate whether the sentence was the first of a passage.

Recall of individual propositions. An analysis was performed on the recall data to determine whether propositions classified as known to individual subjects by the knowledge test were recalled better than propositions that were not known, and whether this effect depended on the task condition. Only those propositions that were actually tested in the knowledge test were included. The proportions of recalled propositions classified by knowledge test score and condition are shown in Table 2. Since data of this type are most appropriately treated as constituting a contingency table, they were analyzed with a three-factor log-linear analysis (Bishop, Fienberg, \& Holland, 1975; Reynolds, 1977), instead of by analysis of variance. The factors were task condition, whether a proposition was known or was not known on the knowledge test, and whether or not the proposition was recalled. The analysis showed that the "saturated" model, which includes all possible main effects and interactions, was necessary to provide a good fit $(p>.05)$ to the data; all lower order models had highly significant differences between the data and the fitted model. This means that the marginal frequencies are different for all three factors (main effects), and that there is statistical dependence between each pair of factors, and between each pair and the third factor (interactions).

The simple main effects are that the probability of recall of a proposition was lower than the probability of

Table 2

Proportion (P) of Recall and Frequency (F) of Previously Known and Unknown Propositions in Each Condition

\begin{tabular}{|c|c|c|c|c|c|c|}
\hline \multirow{3}{*}{$\begin{array}{c}\text { Proposition } \\
\text { Status }\end{array}$} & \multicolumn{6}{|c|}{ Condition } \\
\hline & \multicolumn{2}{|c|}{ Self-Paced } & \multicolumn{2}{|c|}{ Force-Paced } & \multicolumn{2}{|c|}{ Incidental } \\
\hline & $\mathbf{P}$ & $\mathrm{F}$ & $\mathbf{P}$ & $\mathrm{F}$ & $\mathbf{P}$ & $\mathbf{F}$ \\
\hline Unknown & .43 & 1297 & .39 & 1444 & .23 & 1368 \\
\hline Known & .43 & 1553 & .44 & 1406 & .39 & 1482 \\
\hline
\end{tabular}

Note-The proportions are based on the total frequency of the number of known and unknown propositions in each condition. 
nonrecall, that more propositions were known than were not known on the test, and that there was a difference in recall between conditions. Recall was low in the incidental condition, with self-paced and forced-pace recall being roughly the same. The key result is the threefactor interaction, in which the difference in recall between known and unknown propositions differed between conditions. In the self-paced condition, there was no difference in recall between known and unknown propositions. In the forced-pace condition, there was an effect of prior knowledge on recall, with known propositions being recalled somewhat more often than unknown propositions. In the incidental condition, recall of known propositions was very similar to that in the other conditions, but recall of unknown propositions was very low. Thus, known propositions were more likely to be recalled than were unknown ones, and this effect depended on the task.

Sentence-recall regression analysis. The relation between sentence recall and familiarity rating was examined by multiple-regression analyses of each subject's proportion of recall score on each sentence (RECALL) on all four passages in each task. A forward stepwise procedure was used in all regression analyses reported in this paper.

The first step in these analyses was to compute a separate regression analysis for each task. In the selfpaced task, familiarity did not predict recall, replicating the weak effect in the last pilot experiment mentioned above. However, familiarity did predict recall in the forced-pace and the incidental conditions. An analysis of the combined data for all three tasks was then done to test the significance of the interaction of familiarity and condition in predicting recall (see Cohen \& Cohen, 1975, for information on the techniques). The three task conditions were dummy-coded with the variables INCIDENTAL and FORCED, for the incidental and forced-pace tasks, respectively, and with the self-paced task taken as the baseline. The interaction variables FAMINCIDENTAL and FAMFORCED, the product of FAMILIARITY and the corresponding task dummy variable, were defined to represent the contribution of familiarity rating that is distinctive to the incidental and forced-pace tasks. The main-effect variables were forced into the equation first. Note that, in an analysis of this type, the interpretation of a main effect must be qualified by the interaction effect, as in ANOVA.

These results are shown in Table 3 . Table 3 shows the step on which each predictor variable was entered, and the increment in $\mathrm{R}^{2}$ at each step. The coefficients shown are those in the final equation that includes all variables that were entered. The $F$ ratios are the "F-toremove," and thus provide a significance test of the hypothesis that the final coefficient is zero. Finally, the standardized regression coefficients allow comparisons of the importance of each variable independently of scale differences. The final $\mathrm{R}^{2}$ is only .116. One reason for the low percentage of variance accounted for is that the data have not been collapsed into mean reading times for each sentence, as has been customary in analysis of such data (see Anderson, 1982, and Kieras, in press-a).

Notice that the main-effect variable FAMILIARITY is not significant, but that both interaction variables FAMFORCED and FAMINCIDENTAL are significant. This pattern means that a specific form of the task $x$ familiarity interaction is significant. Namely, there is no effect of familiarity in the self-paced task, but familiarity does have an effect in the forced-pace and the incidental tasks. The coefficients for FAMINCIDENTAL and FAMFORCED are not significantly different, suggesting that the effect of familiarity is essentially the same in these two tasks. Familiarity was not a strong variable; its contribution to accounting for variance was quite small, and the coefficients for FAMFORCED and FAMINCIDENTAL show that every point on the familiarity scale adds at most only about $3 \%$ to the percentage of recall.

In order to determine whether the effect of familiarity was due to the possible between-subject differences mentioned above, another analysis was done in which between-subject variability was removed by converting each subject's recall score on the sentences into $\mathrm{z}$ scores computed within each subject, that is, based on

Table 3

Regression Analysis on RECALL for All Tasks

\begin{tabular}{|c|c|c|c|c|c|}
\hline Variable & Step & $\begin{array}{c}\text { Final } \\
\text { Coefficient }\end{array}$ & $\begin{array}{c}\text { Final } \\
\text { Standard Coefficient }\end{array}$ & $\mathbf{R}^{2}$ & $\mathrm{~F}$ \\
\hline CONSTANT & & .219 & & & \\
\hline FAMILIARITY & 1 & .005 & .034 & .0220 & 1.1 \\
\hline INCIDENTAL & 2 & -.216 & -.263 & .0390 & $42.8^{* *}$ \\
\hline FORCED & 3 & -.077 & -.094 & .0391 & $5.8^{*}$ \\
\hline FIRST & 4 & -.189 & -.162 & .0696 & $61,8^{* *}$ \\
\hline WORDS & 5 & -.021 & -.210 & .0962 & $113.3^{* *}$ \\
\hline FREQUENCY & 6 & .010 & .115 & .1062 & $34.9^{* *}$ \\
\hline IMPORTANCE & 7 & -.028 & -.079 & .1109 & $15.8^{* *}$ \\
\hline FAMINCIDENTAL & 8 & .026 & .167 & .1134 & $15.4^{* *}$ \\
\hline FAMFORCED & 9 & .019 & .118 & .1161 & $8.7^{*}$ \\
\hline
\end{tabular}

NOTE $-N=2,880 . \quad * p<.05 . \quad * * p<.01$. 
Table 4

Regression Analysis on Self-Paced-Task Study Times

\begin{tabular}{lccccc}
\hline \multicolumn{1}{c}{ Variable } & Step & $\begin{array}{c}\text { Final } \\
\text { Coefficient }\end{array}$ & $\begin{array}{c}\text { Final } \\
\text { Standard Coefficient }\end{array}$ & $\mathrm{R}^{2}$ & $\mathrm{~F}$ \\
\hline CONSTANT & & 6.552 & & & \\
WORDS & 1 & .355 & .317 & .1231 & $51.2^{*}$ \\
FAMILIARITY & 2 & -.377 & -.227 & .1739 & $94.4^{*}$ \\
FREQUENCY & 3 & -.098 & -.108 & .1812 & $11.4^{*}$ \\
IMPORTANCE & 4 & .382 & .099 & .1905 & $10.9^{*}$ \\
\hline
\end{tabular}

$N O T E-N=960 . \quad * p<.01$.

each subject's mean and standard deviation for recall. This transformation puts all of the subjects on the same scale of recall, removing between-subject differences, but leaving within-subject variation. As reported in detail in Johnson and Kieras (Note 1), the regression on the standardized recall data yields the same predictors and very similar standardized regression coefficients as the analysis on raw RECALL shown in Table 3. Thus, the possible general correlations mentioned above do not account for the role of prior knowledge; it appears within subjects, and apparently has few between-subject components.

As a side result, notice that the coefficients given to FIRST and IMPORTANCE in Table 3 are negative, indicating poorer recall for propositions in the first sentence, which states the main idea, and for sentences rated as being more important. This is consistent with results noted in Kieras (1981b), in which information about highly thematic general items is not necessarily recalled better than less thematic, but more specific, information. However, more detailed analysis would be required to fully explain this effect.

Study time. The multiple-regression analysis in which study time is related to familiarity is shown in Table 4 . This analysis predicts the study time for each subject on each sentence in the self-paced task as a function of the subject's familiarity rating for each sentence and the properties of the sentence itself. These results replicate the last pilot study. The study time for each sentence is predicted by the number of words in the sentence, the familiarity rating, the mean of the Standardized Frequency Index values for the content words in the sentence, and the mean importance rating of each sentence to the main idea. Although an effect of familiarity was present, it was small. Familiarity rating uniquely accounts for only about $4 \%$ of the variance; this is comparable to the results of Graesser, Hoffman, and Clark (1980). Each point on the familiarity scale corresponds to about a .4-sec decrease in study time. About $19 \%$ of the variance in study time is accounted for. As reported in detail in Johnson and Kieras (Note 1), removing the betweensubject variability by z-transforming the study times yielded a very similar set of standardized coefficients. As in the case of the recall results, this shows that the effect of prior knowledge is within subject.

\section{DISCUSSION}

A conclusion from the last pilot experiment was that there was a relationship between familiarity and study time that accounted for a constant level of recall across familiarity. This study reproduced the pilot result, and also demonstrated that recall can be affected by familiarity if the study task is manipulated.

In the self-paced task, the subjects could study unfamiliar sentences for as long as they felt necessary, resulting in the lack of a familiarity effect in recall. The subjects in the forced-pace task, who could not spend additional time on an unfamiliar sentence, recalled familiar material at a higher rate than unfamiliar material. The effects of prior knowledge are strongest in the incidental task, in which recall was $70 \%$ higher for previously known propositions than for previously unknown ones, although the average level of recall was lower than that of the other conditions. These results demonstrate clearly the facilitative effects that prior knowledge should have. It is interesting to speculate that the reason why a clear demonstration has not been reported before is that, since the recall effect is task specific, it may not have appeared in a consistent manner in earlier attempts to produce it.

An important fact was that both the recall and the study-time effects are within subject, as demonstrated by the analyses on z-transformed data reported in Johnson and Kieras (Note 1). This result argues that these effects are genuinely due to the variation in knowledge that each individual reader has about the material, and are not due to general correlations in the subject population.

Another objective of the experiment was to validate the content familiarity ratings as a method of prior-knowledge assessment. Although the correlation between FAMILIARITY and KNOWLEDGE is not extremely high, this level of validity is acceptable for a rating-scale measure. This is important methodologically, since the rating-scale measure is considerably more convenient than the knowledge test as a measure of prior knowledge.

\section{Explaining Familiarity Effects}

The elaboration hypothesis can give a plausible 
account for the pattern of effects of prior knowledge. According to this hypothesis, familiarity with the sentence being studied implies that some redundant retrieval paths for the sentence information should already exist, and more paths should be easy to construct, because there would be a rich supply of related information in memory. Thus, a minimum amount of effort should be needed to encode very familiar material. More processing time should be needed to encode unfamiliar information, because more retrieval paths would have to be built, and a more extensive search of memory would have to be performed to find information that could be used to build elaboration structures.

The success of later recall depends on the amount of elaboration that was accomplished during study. The self-paced task allows the subject to spend as much time as needed to adequately elaborate either familiar or unfamiliar sentences. The forced-pace task does not allow quite enough time for unfamiliar content, and in the incidental task, the subject engages in little elaboration, producing much lower recall. However, notice that in this condition, known information was recalled much better than unknown information and at about the same level as in the other tasks, which presumably require elaboration (see Table 2). If differential elaboration is the sole explanation for prior-knowledge effects, it has to be explained why subjects who are not required to elaborate will recall almost as much on familiar sentences as subjects who are required to elaborate, but will still recall very little on unfamiliar sentences. A possible, but awkward, explanation is that, with familiar material, elaboration is very easy to do, or is even automatic, and thus in the incidental task familiar material is elaborated enough to raise its recall to about the same level as in an intentional task. Unfamiliar material, however, makes elaboration so difficult that very little is done in an incidental task.

The representation-saving hypothesis for the role of prior knowledge also provides a plausible explanation. If a proposition is already known, it need only be tagged, and no new representation need be built in memory. An unknown proposition would take more study time, because the representation would have to be both built and tagged. If this time were restricted, as in the forced-pace task, a lower level of recall for an unknown proposition, compared with a known proposition, results because there is not enough time to complete the encoding process. Similar, and stronger, effects would appear in the incidental task. The subject will not try very hard to build the complete long-term memory representations required for recall, and, therefore, unfamiliar material, which requires both building and tagging, is quite likely to be completely unremembered, whereas familiar material, which only requires the presumably easy tagging process, might be remembered just as well as in an intentional memory task. Notice that this hypothesis seems to be a simpler explanation than the elaboration explanation for the strong effect of prior knowledge in the incidental task, although the data actually do not distinguish them.

Since the representation-saving account of prior knowledge and the elaboration account are compatible, a model incorporating both mechanisms can be offered as an explanation for the effects of prior knowledge. This model states that, as a reader encounters a proposition, a check is performed in long-term memory to see if the proposition is already represented. If it is, it can simply be tagged as seen in this context. If it is not already present in long-term memory, the representation must be built, and then tagged. At this point there should be an increase in processing time for unfamiliar, compared with familiar, material because of the need to build the new representation.

Once a representation has been located or built, and then tagged, elaboration can be done if the subject is processing the propositions for the purpose of later recall. The elaboration process is performed until some criterion of predicted recall success is reached. Familiar material should also take less time in this stage, since there are probably some redundant retrieval paths already present in long-term memory, and there is more related knowledge to support the inference of new paths. If later recall is not expected, the elaboration process is either very cursory, or is not performed. Thus, the recall and processing-time effects of prior knowledge in these results can be attributed to the joint effects of both representation-saving and elaboration. The differences between the intentional and incidental task conditions are a result of the elaboration process's being heavily engaged in intentional tasks, but not in incidental tasks.

\section{A Simulation of Representation Saving}

If an important role of prior knowledge is to simply save on the amount of representation that is to be built, then a simulation model that works on this principle should show a pattern of savings on processing effort that agrees with the data. First, the simulation model will be described briefly, and then how its use of prior knowledge corresponds to the study time data will be described.

The simulation model is described in more detail in Kieras (1977, 1981a, in press-b). This model parses sentences, searches memory for the representations of the given referents (see Haviland \& Clark, 1974), and then adds the new information onto these representations for the given referents. The model has some prior knowledge in its long-term memory and has mechanisms for making use of prior knowledge in constructing representations.

In Kieras (1981a), the amounts of processing of various types in the model were used as predictor variables in a multiple-regression analysis to account for much of the variance in the reading times for sentences in simple passages. More details on this method appear in Kieras (in press-a). Two important types of processing are the 
amount of memory structure that has to be built to represent the sentence content, and the number of parsing steps that have to be done to process the sentence. The amount of memory structure is measured by the number of links of semantic network structure, according to a system based on Anderson's ACT model (Anderson, 1976). The amount of parsing work is measured by the number of transitions that have to be made in the augmented transition network (ATN) used to do the parsing (see Kieras, 1977, in press-b). This work used the same approach as Kieras (1981a), but focused on the effect of prior knowledge on study time. Less memory structure would have to be built if the propositions in a sentence were already known, and the amount of savings of structure should be linearly related to the study time. Thus, the model is a theoretical expression of the representation-saving idea, and the regression fit to the data provided by the model will be examined to see if the representation-saving notion can provide a good account of the data.

The model has three processes for making use of prior knowledge. These processes will identify any portions of memory structure that are already present in the system's long-term memory, and will then use these preexisting representations to reduce the amount of new structure that has to be built. One process searches memory for the representation of a given referent corresponding to a particular individual described in the noun phrase being processed. If a referent is already represented in long-term memory, for example, "the ancient Greeks," this process will find the preexisting representation, and new information can then be added to it. A second process is involved in constructing the representation for a new referent. It searches memory for a concept that is related to the noun phrase that is being processed. A new referent can then be defined in terms of this concept that already exists in long-term memory. For example, this process could find the concept of "religious ceremonies" in long-term memory, and then an instance of this already known concept can be constructed for use in the sentence "The Incas used gold in religious ceremonies." This results in less new structure having to be built. A third process makes use of prior knowledge at the level of the entire sentence, rather than the individual noun phrases. This process examines the propositional structure that needs to be built for the whole sentence after the other two processes have already been applied, and determines whether the new propositions to be added to memory are already represented in long-term memory. It identifies these redundant propositions and removes them from the specifications for the new memory structure.

One approach to using the model would be to give it a set of facts in long-term memory corresponding to the knowledge possessed by an individual subject. Then the model could process each sentence in the passage and report how much parsing work and how much structurebuilding work it had to perform. This could then be repeated for each subject. Since this approach would require considerable computer time, a shortcut was devised, based on the knowledge test results for the individual subjects, which show which individual propositions a subject knew.

Because the parsing work done on each sentence is independent of the amount of prior knowledge, a single processing of each sentence in the passages provided a measure of the amount of parsing work-the number of ATN transitions-required on each sentence (PARSING). By setting up the simulation with no prior knowledge, it could be determined how much memory structurethe number of ACT structure links-had to be built for each sentence when no savings from prior knowledge could be accomplished (TOTAL-STRUCTURE). Exam. ination of the knowledge test scores for each individual subject showed which propositions that person knew and did not know. The known propositions then specified the amount of structure already known by each individual subject (KNOWN-STRUCTURE).

A regression analysis of the study time was then performed; each sentence for each subject had a score corresponding to the amount of memory structure already known by that subject. The total amount of structure for the sentences was also used as a predictor. along with the amount of parsing work for that sentence, both of which did not vary between subjects. The content-word frequency and sentence-importance ratings were also included as predictors. Table 5 shows the results of the regression analysis. The prediction of study time using the simulation variables accounts for $15 \%$ of the variance, which is almost as much as that accounted for when the purely empirical variables are used (Table 4). Notice that the coefficient for the total

Table 5

Regression Analysis on Self-Paced-Task Study Times Using Simulation Variables

\begin{tabular}{|c|c|c|c|c|c|}
\hline Variable & Step & $\begin{array}{c}\text { Final } \\
\text { Coefficient }\end{array}$ & $\begin{array}{c}\text { Final } \\
\text { Standard Coefficient }\end{array}$ & $\mathrm{R}^{2}$ & $\mathbf{F}$ \\
\hline CONSTANT & & 7.382 & & & \\
\hline PARSING & 1 & .028 & .212 & .0812 & $15.5^{* *}$ \\
\hline KNOWN-STRUCTURE & 2 & -.049 & -.206 & .1083 & $36.8 * *$ \\
\hline TOTAL-STRUCTURE & 3 & .068 & .235 & .1312 & $17.9 * *$ \\
\hline FREQUENCY & 4 & -.140 & -.154 & .1464 & $20.0^{* *}$ \\
\hline IMPORTANCE & 5 & .290 & .075 & .1513 & $5.5^{*}$ \\
\hline
\end{tabular}

NOTE $-N=960 . \quad{ }^{*} p<.05 . \quad{ }^{* *} p<.01$. 
amount of structure is roughly similar in size to the coefficient for the already known structure, but the known structure coefficient is negative. Hence, study time can be predicted from the difference between the total amount of structure and the amount of known structure, or simply the amount of new unknown structure.

The conclusion is that, since the model's representation of prior knowledge can be used to account for some of the variance in the results, the representationsaving idea is a reasonable representation for at least part of the effect of prior knowledge on comprehension and memory.

\section{Relation to Schema Theory}

Although the above arguments and results downplay the role of schemata in technical prose, they should not be seen as incompatible with schema theories in general. In prose for which a reader has a relevant schema, the search of memory for a previously known proposition should proceed faster, because the schema should guide the search efficiently. If a schema is applicable to the material, many of the propositions may already be implicitly or explicitly represented in the schema itself, reducing the amount of structure to be built and providing many elaborative interconnections as well. Hence, at least some of the facilitative effects of prior knowledge at the schema level could actually consist of effects at the levels of elaboration and representation saving.

\section{The Weakness of Familiarity Effects}

An important final conclusion is that the effect of prior knowledge in these results is not very strong. The proportion of variance accounted for by prior knowledge is fairly low, only a few percent. This seems counterintuitive. The materials produced drastic differences in the amount of information individual subjects already knew. However, there is no corresponding drastic difference in study time, and there is not much difference in probability of recall in an intentional recall task. It would certainly seem obvious that if one already knows the material, very little effort should be required to memorize it, and recall should be very good. This is the obvious intuition, but the intuition seems to be false. How can this weak effect of prior knowledge be explained?

One possibility is that the procedure of presenting the sentences twice simply attenuated the effect of prior knowledge. But, as argued above in the last pilot study, there is no clear indication that this is so. Another possibility is that the prior knowledge manipulation was not strong enough. However, the size of the effect is compatible with that reported by Graesser, Hoffman, and Clark (1980), and, intuitively, the passage facts covered the familiarity range very effectively.

Perhaps the best explanation for the weakness of familiarity effects is based on the nature of the prose memory paradigm and how a typical subject will ap- proach it. In prose memory experiments, subjects are asked to study and then to recall within a short span of time, roughly $1 \mathrm{~h}$. It could be that a typical subject, being a college student, is very good at using powerful elaboration strategies for memorizing information that is not very familiar in order to recall it in the near future. If subjects have this skill, the result would be a tendency for unfamiliar material to be recalled very similarly to familiar material, swamping the strong familiarity difference that should be there. This hypothesis is confirmed by the strong prior-knowledge effect on recall in the incidental tasks, in which, presumably, this type of encoding strategy is not engaged.

This conclusion suggests that in order to study the role of prior knowledge in comprehension, tasks must be used that do not engage such strategies. Perhaps there are some ecologically valid reading and memory tasks that are strongly dependent on prior knowledge, but it appears that the standard prose memory task is not such a task.

\section{REFERENCE NOTES}

1. Johnson, W., \& Kieras, D. E. The role of prior knowledge in the comprehension of simple technical prose (Tech. Rep. UARZ/ DP/TR-82/ONR-11). Tucson, Ariz: University of Arizona, Department of Psychology, April 1982.

2. Bovair, S., \& Kieras, D. E. A guide to propositional analysis for research on technical prose (Tech. Rep. 8). Tucson, Ariz: University of Arizona, Department of Psychology, July 1981.

\section{REFERENCES}

ANderson, J. R. Language, memory, and thought. Hillsdale, N.J: Erlbaum, 1976.

Anderson, J. R. Effects of prior knowledge on memory for new information. Memory \& Cognition, 1981, 9, 237-246.

Anderson, J. R. Allocation of attention during reading. In A. Flammer \& W. Kintsch (Eds.), Discourse processing. Amsterdam: North-Holland, 1982.

Anderson, J. R., \& Bower, G. H. Human associative memory. Washington, D.C: Winston, 1973.

Anderson, J. R., \& Reder, L. M. An elaborative processing explanation of depth in processing. In L. S. Cermak, \& F. I. M. Craik (Eds.) Levels of processing in human memory. Hillsdale, N.J: Erlbaum, 1979.

Bishop, Y. M. M., Fienbera, S. E., \& Holland, P. W. Discrete multivariate analysis: Theory and practice. Cambridge, Mass: MIT Press, 1975.

Carroll, J. B., Davies, P., \& Richman, B. American Heritage word frequency book. New York: American Heritage, 1971.

Chiesi, H. L., Spilich, G. J., \& Voss, J. F. Acquisition of domain related information in relation to high and low domain knowledge. Journal of Verbal Learning and Verbal Behavior, $1979,18,257-273$.

Cohen, J., \& CohEn, P. Applied multiple regression/correlation analysis for the behavioral sciences. Hillsdale, N.J: Erlbaum, 1975.

GaGne, E. D. Long-term retention of information following learning from prose. Review of Educational Research, 1978, 48, 629-665.

Graesser, A. C. Prose comprehension beyond the word. New York: Springer-Verlag, 1981.

Graesser, A. C., Hauft-Smith, K., Cohen, A. D., \& Pyles, L. D. Advanced outlines, familiarity, and text genre on retention of prose. Journal of Experimental Education, 1980, 40, 281-290. 
Graessen, A. C., Hoffman, N. L., \& Chark, L. F. Structural components of reading time. Journal of Verbal Learning and Verbal Behavior, 1980, 19, 135-151.

Haviland, S. E., \& Clark, H. H. What's new? Acquiring new information as a process in comprehension. Journal of Verbal Learning and Verbal Behavior, 1974, 13, 512-521.

Johnson, R. E. Meaningfulness and the recall of textual prose. American Educational Research Journal, 1973, 10, 49-58.

Kis ras, D. E. Problems of reference in text comprehension. In M. Just \& P. Carpenter (Eds.), Cognitive processes in comprehension. Hillsdale, N.J: Erlbaum, 1977.

Kieras, D. E. Component processes in the comprehension of simple prose. Journal of Verbal Learning and Verbal Behavior, $1981,20,1-23$. (a)

KIERAS, D. E. Topicalization effects in cued recall of technical prose. Memory \& Cognition, 1981, 9, 541-549. (b)

KIERAs, D. E. A model of reader strategy for abstracting main ideas from simple prose. Text, 1982, 2, 47-82.

KiEras, D. E. A method for comparing a simulation model to reading time data. In D. Kieras \& $\mathrm{M}$. Just (Eds.), New methods in comprehension research. Hillsdale, N.J: Erlbaum, in press. (a)

KIERAs, D. E. A simulation model for the comprehension of technical prose. In G. H. Bower (Ed.), The psychology of learning and motivation (Vol. 17). New York: Academic Press, in press. (b)

KiERAs, D. E. Thematic processes in the comprehension of tech- nical prose. In B. Britton \& J. Black (Eds.), Understanding expository prose. Hillsdale, N.J: Erlbaum, in press. (c)

KInTsCh, W., \& van DiJK, T. A. Toward a model of discourse comprehension and production. Psychological Review, 1978, 85, 363-394.

Paivio, A. Imagery and verbal processes. New York: Holt, Rinehart \& Winston, 1971.

REYNOLDs, H. T. The analysis of cross-classifications. New York: Free Press, 1977.

Rumelhart, D. E. Schemata: The building blocks of cognition. In R. Spiro, B. Bruce, \& W. Brewer (Eds.), Theoretical issues in reading comprehension. Hillsdale, N.J: Erlbaum, 1980.

Rumelhart, D. E., \& Ortony, A. The representation of knowledge in memory. In R. C. Anderson, R. J. Spiro, \& E. Montague (Eds.), Schooling and the acquisition of knowledge. Hillsdale, N.J: Erlbaum, 1977.

Spilich, G. J., Vesonder, G. T., Chiesi, H. L., \& Voss, J. F. Text processing of domain related information for individuals with high and low domain knowledge. Journal of Verbal Learning and Verbal Behavior, 1979, 18, 275-290.

ThonNDYke, P. W. Cognitive structures in comprehension and memory of narrative discourse. Cognitive Psychology, 1977, 9, 77-110.

(Manuscript received November 5, 1982; revision accepted for publication April 27, 1983.) 